\title{
Automatische Wiedererkennung von individuellen Objekten mit dem Dynamic Anchoring Agent
}

\author{
Martin Günther $\mathbb{D} \cdot$ Friedemann Kammler $(\mathbb{D} \cdot$ Oliver Ferdinand $(\mathbb{D} \cdot$ \\ Joachim Hertzberg $\mathbb{D} \cdot$ Oliver Thomas $\cdot$ Oliver Zielinski $\mathbb{D}$
}

Eingegangen: 30. Juni 2020 / Angenommen: 10. Oktober 2020 / Online publiziert: 28. Oktober 2020

(C) Der/die Autor(en) 2020

Zusammenfassung Individuelle Objekte spielen im Alltag eine zentrale Rolle und machen deren Unterscheidung zu einer wichtigen Voraussetzung für den Einsatz von Robotik. Die Herausforderung liegt dabei nicht allein in der sensorischen Wahrnehmung, sondern auch in der Verknüpfung mit vorhandenem Wissen: So können zwei für Verfahren der Objekterkennung identische Gegenstände durch ihre Funktion (,Der Akkuschrauber, den ich zuletzt genutzt habe“) oder ihren Umgebungskontext („Die Tasse auf meinem Tisch“) eine eindeutige Zuordnung erhalten. Die Robotik adressiert diese Herausforderung unter dem Begriff des Anchorings, also der Fähigkeit, individuelle Objekte eindeutig zu erkennen und anhand ihres Kontexts wiederzuerkennen, sobald sie einmal aus dem Wahrnehmungsbereich gelangt sind. Auf technischer Ebene besteht dabei das Problem, Beziehungen zwischen Sensordaten und Symbolen in einer Wissensbasis herzustellen und so physische Objekte konkret zu adressieren. Der Beitrag stellt das Anchoring-Problem sowie den Dynamic Anchoring Agent (DAA) als einen Lösungsansatz vor. Anhand von zwei realen Anwendungsszenarien wird der Einsatz des DAA demonstriert: Anhand eines MakerSpaces werden die Möglichkeiten zur erweiterten Kooperation zwischen

\footnotetext{
M. Günther · J. Hertzberg

Deutsches Forschungszentrum für Künstliche Intelligenz GmbH (DFKI), Planbasierte

Robotersteuerung, Berghoffstraße 11, 49080 Osnabrück, Deutschland

F. Kammler $(\bowtie) \cdot$ O. Thomas

Deutsches Forschungszentrum für Künstliche Intelligenz GmbH (DFKI), Smart Enterprise

Engineering, Parkstraße 40, 49080 Osnabrück, Deutschland

E-Mail: friedemann.kammler@dfki.de

O. Ferdinand · O. Zielinski

Deutsches Forschungszentrum für Künstliche Intelligenz GmbH (DFKI), Marine Perception,

Marie-Curie-Straße 1, 26129 Oldenburg, Deutschland

O. Thomas

Fachgebiet Informationsmanagement und Wirtschaftsinformatik, Universität Osnabrück, Parkstraße 40, 49080 Osnabrück, Deutschland
} 
Menschen und Robotern gezeigt - beispielsweise durch die Suche und Identifikation von persönlichem Werkzeug oder benötigten Produktionsmaterialien. Eine zweite Anwendung verdeutlicht am Beispiel eines Yachthafens den weiterführenden Einsatz in dynamischen Umgebungen.

Schlüsselwörter Maschinelles Lernen · Anchoring Problem · Dynamic Anchoring Agent $\cdot$ Yachthafen $\cdot$ MakerSpace $\cdot$ Mensch-Maschine-Kooperation

\title{
The Dynamic Anchoring Agent for Automatic Identification of Individual Objects
}

\begin{abstract}
Individual objects play a central role in everyday life and make their distinction an important requirement for the application of robotics. The challenge is not only sensorial perception, but also the combination with existing knowledge: For example, two goods that are identical for object recognition can be further distinguished by their function ("The battery screwdriver I used last") or their environmental context ("The cup on my table"). Robotics addresses this challenge under the notion of anchoring, meaning the ability to identify individual objects and to recognize them by their context once they have left the perception of the system. On a technical level, the problem is to establish relationships between sensor data and symbols in a knowledge base and thus to address physical objects concretely. This paper presents the anchoring problem and the Dynamic Anchoring Agent (DAA) as a solution approach. The use of the DAA is demonstrated using two real application scenarios: With the example of a MakerSpace, the possibilities for extended cooperation between humans and robots are shown-for instance in the search and identification of personal tools or required production materials. The second application demonstrates the extended use in dynamic environments within the example of a marina.
\end{abstract}

Keywords Machine Learning - Anchoring Problem - Dynamic Anchoring Agent · Marina $\cdot$ MakerSpace $\cdot$ Human-Machine-Cooperation

\section{Kooperative Zusammenarbeit von menschlicher und maschineller Intelligenz}

„Roboter, bring mir meinen Akkuschrauber!“ - so oder so ähnlich könnte ein plausibler Befehl für einen Serviceroboter in einer Produktionsumgebung oder im Privathaushalt lauten. Roboter, die ein Werkzeug an einen Menschen übergeben, wurden vielfach demonstriert - zum Beispiel auf dem Stand des Deutschen Forschungszentrums für Künstliche Intelligenz (DFKI) auf der Hannover Messe 2019 (vgl. Abb. 1). Die Fähigkeit, Objekte zu erkennen und zu bringen, ist mittlerweile Stand der Technik. Wie aber ist der Fortschritt zu bewerten, wenn nicht nur irgendein Akkuschrauber oder ein bestimmter Typ identifiziert werden soll, sondern eindeutige Zuordnungen (,mein“ Akkuschrauber) zu beachten sind? 
Abb. 1 Umgang mit einem individuellen Objekt - oder doch nicht? Bereitstellung eines Akkuschraubers durch einen Roboter: Demo im Rahmen der Hannover Messe 2019 am Stand des DFKI

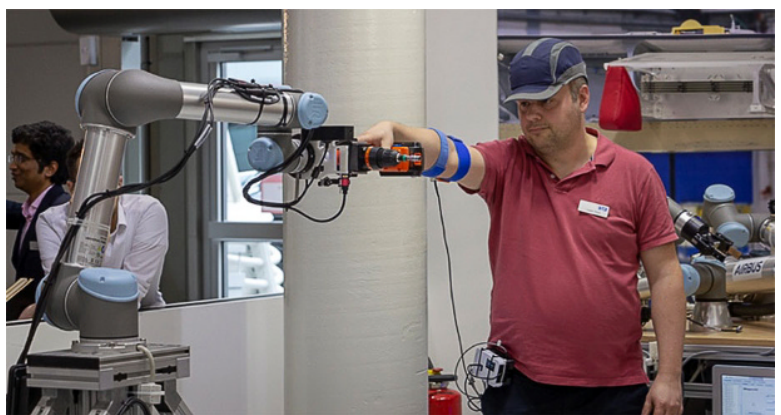

Damit ein Serviceroboter in einer alltäglichen Umgebung umfassend einsetzbar ist, muss er in der Lage sein, nicht nur Objektklassen, sondern auch individuelle Objekte zu unterscheiden und über längere Zeit eindeutig zuzuordnen. Man beachte, dass es dabei nicht nur um die Erkennung aus Sensordaten geht: Wie beim Akkuschrauber (und bei Geschirrteilen auf einem festlich gedeckten Tisch, bei einer Tasse im Büro und vielem mehr) umgeben uns viele identisch aussehende oder sehr ähnliche Objekte, deren Individualität funktional definiert ist (,der Akkuschrauber, den ich zuletzt benutzt habe“) und in vielen Fällen dem räumlichen Kontext entspricht (,die Tasse auf meinem Schreibtisch“). Hierdurch entstehen umfassendere Herausforderungen für die Robotik, denn eine ontologische Repräsentation mit einer Reihe von objektiv verschiedenen Einzelobjekten (Tasse-21, Tasse-22, Tasse23), die identisch aussehen, hilft nur dann weiter, wenn man davon ausgeht, dass jedes einzelne Objekt diese einzigartige Identität als Signatur eingraviert hat (z.B. durch einen RFID-Tag) und nicht wieder verliert.

Derartige Szenarien existieren bereits in Laborumgebungen, erscheinen jedoch für viele alltägliche Servicefälle nicht realistisch. Denn einerseits entsteht ein hoher Initialaufwand, eindeutige Signaturen zu erstellen, andererseits ist der Aufwand für deren Bereitstellung praktisch vergeudet, da wir uns meist nicht um die objektive Identität verschiedener, gleich aussehender Instanzen von Dingen kümmern, sondern nur um die funktionale Identität für einen bestimmten Zweck: Ich interessiere mich für „,meine Tasse“, die im Moment und für den Rest des Tages „Tasse-22“ ist. Diese funktionale Identität ist verschwunden, wenn die Tasse am Ende des Tages in die Spülmaschine kommt, und ich würde normalerweise nicht verlangen, die objektiv identische, individuelle Tasse am nächsten Tag wieder zu benutzen - es sei denn, sie hat einige einzigartige Eigenschaften, die sie zu „meiner Lieblings-Tasse überhaupt“ machen. In diesem Fall würde sie sich jedoch auch sensorisch von all den anderen Tassen unterscheiden.

Selbstverständlich ist die Vorstellung von einem Serviceroboter im Haushalt noch Zukunftsmusik. Jedoch eröffnen Fortschritte in der Robotik und Künstlichen Intelligenz jetzt schon Einsatzfelder für Roboter jenseits der klassischen Automatisierung. Beispielsweise werden bereits von mehreren Startups (z. B. Bossa Nova Robotics, Simbe Robotics, Zebra Technologies) Roboter entwickelt, die in Supermärkten Produkte inventarisieren, die Preisausschilderung auf Korrektheit überprüfen und falsch einsortierte Produkte erkennen können. Der Schritt von einer hochstrukturierten und 
-kontrollierbaren Fabrikumgebung, wie sie für klassische Automatisierungstechniken erforderlich ist, hin zur hochdynamischen Umgebung eines Supermarkts wurde durch Fortschritte in der Robotik (z. B. sichere Navigation und Hindernisvermeidung in Bereichen mit Publikumsverkehr, Pütz et al. 2018), Hardware (z. B. günstige und präzise 3D-Kameras, Grenzdörffer et al. 2020) und Künstlichen Intelligenz (z.B. Objekterkennung mit Künstlichen Neuronalen Netzen, Deep Learning, Tremblay et al. 2018) ermöglicht. Gleichzeitig erfordert die Supermarktdomäne kein Zuordnen von individuellen Objekten; alle Artikel eines Typs können gleichbehandelt werden. In anderen Anwendungsbereichen (z. B. Produktion und Wartung in Manufakturbetrieben, Elektroniklaboren, Werkstätten oder MakerSpaces) ist es jedoch sehr wohl notwendig, individuelle Objekte derselben Klasse (z. B. Werkzeuge oder Werkstücke) eindeutig zuzuordnen. Dies ist das sogenannte Anchoring-Problem, und zu der Lösung dieses Problems soll der Dynamic Anchoring Agent (DAA) einen Beitrag leisten.

Anhand von zwei realen Anwendungsszenarien wird der Einsatz des DAA demonstriert: Eine Produktionsumgebung verdeutlicht die Möglichkeiten zur erweiterten Kooperation zwischen Menschen und Robotern - beispielsweise durch die Suche und Identifikation von persönlichem Werkzeug oder benötigten Produktionsmaterialien (vgl. Abb. 1). Komplementär zeigt das zweite Szenario die generelle Verwendbarkeit auch in Außenszenarien, indem der DAA Informationen aus Sensorstationen, die über eine Hafenanlage verteilt sind, bezieht und zur umfassenden Wahrnehmung der Hafenbewegungen nutzt.

Die Entwicklung des DAA ist Teil des laufenden Forschungsprojekts CoPDA am Deutschen Forschungszentrum für Künstliche Intelligenz (DFKI). Sobald der DAA eine ausreichend weitgehende und stabile Version erreicht hat, wird er der Öffentlichkeit als Softwarepaket für das Robot Operating System (ROS) zur Verfügung gestellt werden.

\section{Das Anchoring-Problem}

$\mathrm{Zu}$ erkennen, welches „meine Tasse“ ist, ist für uns Menschen trivial: Wir kommen damit ohne kognitive Anstrengung im täglichen Leben klar. In der Servicerobotik ist diese Fähigkeit zum Umgang mit individuellen Objekten praktisch ohne Beispiel. Sie erfordert die Verankerung (Anchoring), das heißt den Prozess der „Erstellung und Erhaltung der Korrespondenz zwischen Symbolen und Sensordaten, die sich auf dieselben physikalischen Objekte beziehen" (Coradeschi und Saffiotti 2003). Das Verankerungsproblem hat in der wissenschaftlichen Literatur Aufmerksamkeit erlangt, ist aber noch lange nicht gelöst oder gar vollständig verstanden. Verankerung ist aufgrund seiner Verortung an der kaum erforschten Schnittstelle von Robotik und Wissensrepräsentation und -verarbeitung als schwieriges Problem anzusehen, das feldübergreifende Ansätze zum Verständnis und - im besten Fall - zur erfolgreichen Bewältigung erfordert.

Eine robuste Lösung des Verankerungsproblems ist entscheidend für alle Operationen von hybriden Mensch-Roboter-Teams in unvollständig kontrollierten, dynamischen und/oder unvollkommen erfassten Umgebungen, die sich über den Raum 
und über längere Zeit erstrecken. Wann immer ein individuelles Objekt oder ein bestimmtes Objekt einer Menge nur aus Sensordaten und Vorwissen über das Objekt in Raum und Zeit erkannt werden muss, müssen wir ein Verankerungsproblem lösen. Das konzeptionelle Problem besteht darin, die Korrespondenz zwischen dem physischen Objekt und seiner Repräsentation über einen „Anker“ effektiv aufrechtzuerhalten, der die Repräsentation dieser Korrespondenz ist und daher seinerseits ein Gegenstand von Schlussfolgerung und Lernen sein kann. Die Verfolgung (Tracking) eines Objekts in der realen Welt aus verrauschten und lückenhaften Sensordaten ist ein typischer Fall des Problems; andere resultieren aus unabhängiger Domänendynamik, wie beispielsweise das Wiederfinden eines Objekts, das sich aktiv bewegt hat, das passiv im Raum verschoben wurde oder das sich faktisch im Erscheinungsbild verändert hat, seit es zuletzt wahrgenommen wurde (wie eine Tasse bei veränderter Beleuchtung oder eine Yacht mit gesetzten Segeln). Mit einer praktischen Verankerungsmethode wäre es einem Serviceroboter endlich möglich, mir meine Tasse oder meinen Akkuschrauber zu bringen - und nicht irgendeine oder die einzige weit und breit verfügbare, wie wir das aus so vielen Hol-und-Bring-Demos kennen.

Wahrnehmungsdaten, die sich auf physische Objekte und entsprechende abstrakte Darstellungen in symbolischen Repräsentationen beziehen, werden durch den Prozess der Verankerung verknüpft (Coradeschi und Saffiotti 2003). Verankerung ist damit ein spezifisches Beispiel des allgemeinen Symbol Grounding Problems (Harnad 1990), das sich für Roboter stellt, die ihre physische Umgebung wahrnehmen (Coradeschi et al. 2013).

Die Robotik/KI-Literatur seit Anfang der 2000er-Jahre enthält etliche Ansätze zur Bewältigung des Problems (vgl. Überblick in Coradeschi et al. 2013). In jüngerer Zeit wurden mehrere probabilistische Ansätze zur Kombination von Objekt- und Sensorinformationen veröffentlicht (z. B. Blodow et al. 2010; Elfring et al. 2013; Wong et al. 2015; Günther et al. 2018; Persson et al. 2019). Das Grundproblem enthält jedoch viele ungelöste Facetten; insbesondere bei Forschungsarbeiten befassen sich die meisten veröffentlichten mit Laborumgebungen (,Table-Top-Robotik“), mit all ihren Vor- und Nachteilen, aber wenden die Konzepte und Teillösungen der Verankerung nicht auf reale Anwendungen an. Anchoring (und semantische Kartierung (Deeken et al. 2019) im Allgemeinen) bietet gewisse Überschneidungen mit traditionellem Multi-Target-Tracking und Datenassoziation. Es gibt jedoch mehrere wesentliche Unterschiede (Wong et al. 2015):

- Objekte haben neben dem Ort weitere Attribute und es gelten semantische Relationen zwischen ihnen,

- nur ein kleiner Ausschnitt der Welt befindet sich zu jedem Zeitpunkt im Sichtfeld des Roboters, während die meisten Tracking Ansätze davon ausgehen, dass alle Ziele zu jedem Zeitpunkt erfasst werden und

- Objekte bleiben über kurze Zeiträume typischerweise stationär, so dass das Frameto-Frame-Tracking weniger problematisch ist als die Verankerung eines Objekts zwischen Beobachtungen, die durch längere Zeitspannen getrennt sind.

Während die Verankerung über die Datenassoziation hinausgeht, sind viele der Techniken nach wie vor anwendbar und können in eine Verankerungsmethode integriert werden. Ein verbreiteter Basisalgorithmus zur Datenassoziation ist der Global 
Nearest Neighbor (GNN) Algorithmus (Bar-Shalom et al. 2011), den die Autoren in früheren Arbeiten selbst verwendet haben (Günther et al. 2018). Die prominentesten Vertreter höher entwickelter Alternativen zum GNN sind der Joint Probabilistic Data Association Filter (JPDAF; Blom und Bloem 2002) und Approximationen des Multiple Hypothesis Tracking (MHT; Reid 1979). Diese Algorithmen stellen die Entscheidung zurück, welches Objekt welchem Symbol zugeordnet werden soll und verwenden Informationen aus späteren Frames, um die Zuordnung eindeutig zu machen. Dies verbessert die Zuordnungsgenauigkeit insbesondere in Situationen mit vielen störenden Objekten, in denen GNN unter seiner lokalen Zuordnungsstrategie leidet und häufig Symbole vorschnell einem falschen Objekt zuordnet. MHT wurde bereits mit Erfolg für Verankerung eingesetzt (Elfring et al. 2013) und bildet die Grundlage der Verankerungsmethodik des hier vorgestellten CoPDA-Projekts.

Die Interaktion zwischen Akteuren und DAA wird mittels einer eigens entwickelten Mensch-Maschine-Schnittstelle (engl. Human-Machine Interface, HMI) realisiert. Bisherige Forschungsarbeiten liefern bereits zahlreiche Anknüpfungspunkte zum Design oder der Implementierung solcher Schnittstellen. Gleichzeitig führt die zunehmende Verknüpfung von Objekten, wie sie unter dem Begriff Industrie 4.0 diskutiert werden, zu einer Veränderung in der Bedeutung und Rolle des Menschen. Gorecky et al. (2014) beschreiben die Notwendigkeit, relevante Informationen kontextsensitiv bereitzustellen, um den Menschen in solchen Kontexten zu unterstützen. Bei der Einführung neuer Technologien an der Schnittstelle zwischen Menschen und Maschine, wie sie bspw. mit Augmented-Reality-Brillen erprobt wird, bleibt zu prüfen, wie diese den Menschen in seinen Aktivitäten bereichern kann (Kammler et al. 2019). Verankerung könnte sich als wesentlicher Bestandteil erweisen, um dem Menschen die erforderlichen Kontextinformationen in Umgebungen mit einzelnen Objekten zur Verfügung zu stellen.

\section{Der Dynamic Anchoring Agent}

\subsection{Architektur}

Das Projekt CoPDA entwickelt mit dem Dynamic Anchoring Agent eine Software, die in der Lage ist, das Verankerungsproblem unter bestimmten Bedingungen zu lösen. Dieser DAA, der auf einer speziellen Wissensrepräsentation und einer speziellen (Multi-)Sensorkonfiguration basiert, wird in verschiedenen Umgebungen konzipiert, implementiert, getestet und demonstriert, einschließlich eines Innen- und eines Außeneinsatzes (Produktionsumgebung und Yachthafen). Da die Verankerung als solche bislang keine etablierten Methoden aufweist, greifen wir hierfür auf die wegweisende Arbeit von Coradeschi und Saffiotti (2003) zurück, die drei grundlegende Verankerungsfunktionen einführt:

- Finden ( find) der Zuordnung eines Objekts zu einem bereits vorhandenen Symbol in der Repräsentation in Sensordaten, gegeben eine Erwartung der Erscheinung des Objekts in den Sensordaten, 
- Verfolgen (track) des Aufenthaltsortes eines verankerten Objekts unter Beobachtung und

- Wiederfinden (reacquire) eines bereits verankerten Objekts, das außerhalb des Sensorbereichs lag und das aufgrund der Kenntnis seines früheren Aufenthaltsortes, seines Erscheinungsbildes und seiner erwarteten Dynamik während der Zeit der Nichtbeobachtung neu identifiziert werden muss.

Während mindestens diese Funktionen zur Verankerung benötigt werden, ist schnell ersichtlich, dass sie nicht für alle praktischen Zwecke ausreichen. Beispiele sind die Verankerung neuer Objekte, die bisher nicht repräsentiert waren (wie in der semantischen Kartierung), die Verankerung von Objektsymbolen mit zeitlich veränderlichen Denotaten (wie oben ,meine Tasse“) und die Verankerung von Objekten, die auf Relationen basieren (wie qualitative räumliche Relationen, die mir helfen, in einem Dinner-Szenario festzustellen, was „meine Tasse“ sein soll). Ein weiterer wichtiger Aspekt ist die Entscheidung, welche wahrgenommenen Objekte überhaupt verankert werden sollen (mein Glas auf einer Party - ja; alle anderen Gläser - nein) und für welches zuvor verankerte Objekt der Anker ,gelichtet“ werden soll (wie meine Tasse, wenn sie in die Spülmaschine kommt). Die Herausforderung besteht darin, einen geeigneten Satz von Verankerungsfunktionen für den DAA zu identifizieren, der sowohl dem Zuschnitt des Projekts als auch den Demonstrationsszenarien entspricht.

Die interne Softwarearchitektur des Dynamic Anchoring Agents sowie seine Einbettung in die größere Roboterarchitektur ist in Abb. 2 dargestellt. Der Weltzustand ist hier als der tatsächliche Zustand der realen Umgebung des Roboters zu verstehen. Die Wahrnehmung ist Teil der Robotersteuerungssoftware. Ausgehend von den Sensordaten (z. B. Bilder, Tiefenbilder oder Laserscans) liefert ein Neuronale-Netzbasiertes Objekterkennungsverfahren die Klassen (z.B. „Tasse“ oder „Segelboot“) und die dynamischen Attribute (z. B. „Füllstand“ oder „Segel gesetzt“) der Objekte. Diese sogenannten Percepts enthalten noch keine Verknüpfung zu Objektidentitäten; außerdem sind sie lediglich fehlerbehaftete und unvollständige Beobachtungen des tatsächlichen (nicht direkt beobachtbaren) Weltzustands.

Die Aufgabe der Weltmodellierung in Form des Dynamic Anchoring Agents besteht darin, den Weltzustand möglichst gut aus den Percepts zu approximieren. Dazu verwendet der DAA eine Variante des Multi-Hypothesis-Trackings (MHT; Reid 1979; Elfring et al. 2013): Für jedes neue Percept gibt es üblicherweise mehrere plausible Möglichkeiten, mit welchem bekannten Symbol es verankert werden kann; weiterhin könnte es zu einem bislang nicht beobachteten Objekt gehören, sodass ein neues Symbol erzeugt werden müsste. Im Multi-Hypothesis-Tracking-Ansatz wird diese Entscheidung nicht sofort getroffen, sondern zurückgestellt, indem für jede plausible Zuordnung eine neue Hypothese angelegt wird. Eine Hypothese spiegelt dabei immer einen vollständigen möglichen Weltzustand in Form von Symbolen und ihren Eigenschaften wie Position, Orientierung, Objektklasse und dynamischen Attributen wider; diese Eigenschaften werden durch die Fusionierung der zugeordneten Percepts geschätzt. Alle aktuell verfolgten Hypothesen werden im Hypothesenspeicher vorgehalten. 
Auf diesem Hypothesenspeicher arbeiten zwei Teilagenten des DAA: Der Probabilistic Reasoning Agent und der Logic Reasoning Agent. Der Probabilistic Reasoning Agent erzeugt (wie oben beschrieben) Hypothesen und fügt sie zum Hypothesenspeicher hinzu. Weiterhin berechnet er für jede Hypothese eine Wahrscheinlichkeit $p$, basierend auf einem probabilistischen Modell der Zuordnung von Objekten. Beispielsweise ist eine Hypothese, die aus der Zuordnung von Symbol $S$ zu Percept $P$ entstanden ist, um so wahrscheinlicher, je ähnlicher sich das Erscheinungsbild von $P$ und den aktuell geschätzten Eigenschaften von $S$ sind. Um der beliebigen Expansion des Hypothesenraums entgegenzuwirken, werden jederzeit nur die wahrscheinlichsten $n$ Hypothesen gespeichert (typischerweise wenige hundert); alle unwahrscheinlicheren werden verworfen. Die zurzeit wahrscheinlichste Hypothese wird MaximumA-Posteriori-Hypothese (MAP-Hypothese) genannt.

Der zweite Teilagent ist der Logic Reasoning Agent. Er arbeitet ebenfalls auf dem Hypothesenspeicher und prüft die Hypothesen auf logische Konsistenz. Dazu werden die Hypothesen mit einer Wissensbasis in Form des Domänenmodells verglichen, in dem in Form von logischen Regeln das Wissen über den Prozess abgelegt wird. Logisch mit dem Domänenmodell inkonsistente Hypothesen werden verworfen.

Die Handlungsplanung reagiert auf Ziele, die vom Anwender vorgegeben werden (,Bring mir meinen Akkuschrauber!“). Auf Grundlage von statischem Wissen über die möglichen Aktionen und ihre Effekte, welche im Domänenmodell hinterlegt sind, sowie dem damit verankerten dynamischen Wissen über die bekannten Objekte und ihre Identitäten aus der MAP-Hypothese wird ein Plan (eine Sequenz von Aktionen) erzeugt, der anschließend vom Roboter ausgeführt wird. Der Einsatz

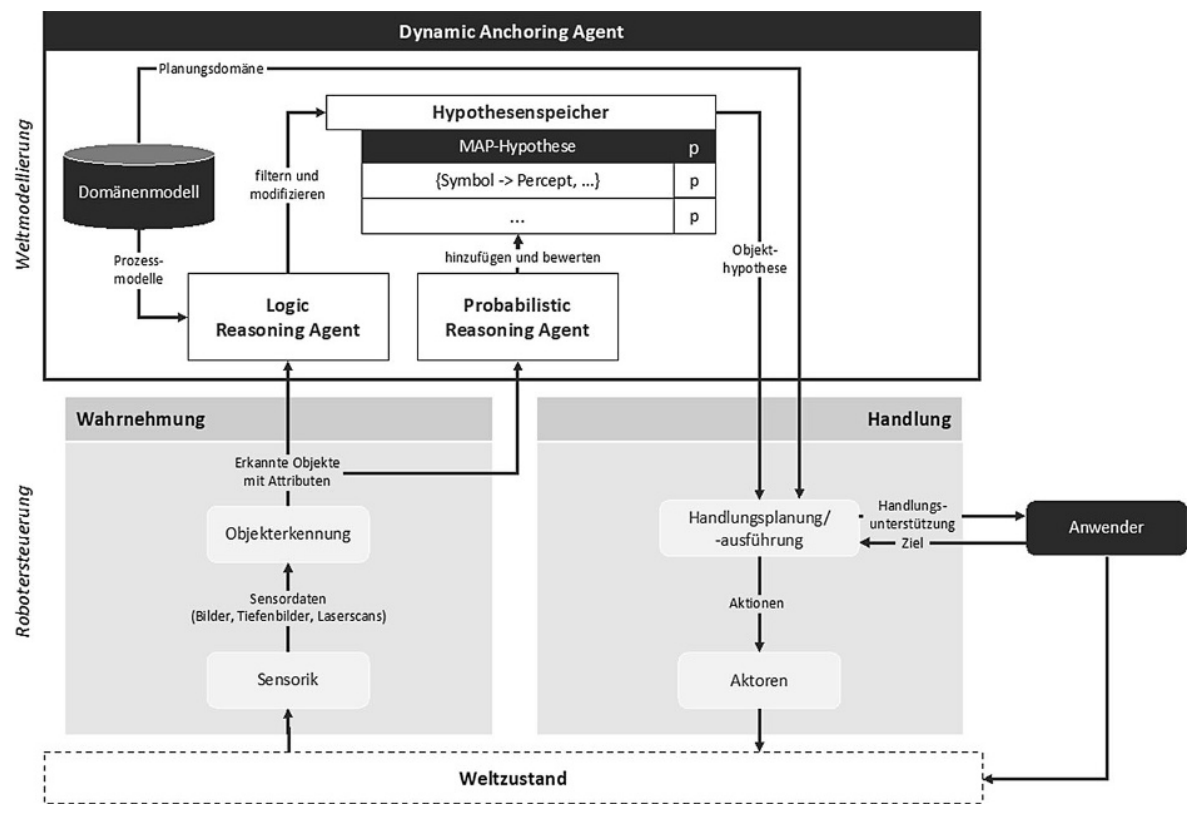

Abb. 2 Softwarearchitektur des Dynamic Anchoring Agents 
des DAA wird nachfolgend anhand der zwei Demonstrationsszenarien des CoPDAProjekts illustriert.

\subsection{Der MakerSpace als unstrukturierte Produktionsumgebung}

Der praktische Nutzen des DAA für Produktionsumgebungen zeigt sich vor allem in wenig strukturierten und dynamischen Umgebungen, wie am Beispiel von MakerSpaces anschaulich demonstriert werden kann. MakerSpaces folgen dem PeerProduction-Ansatz und stellen in offenen Werkstätten Produktionsmittel (Werkzeug, Maschinen und Arbeitsfläche) zur kooperativen Nutzung zur Verfügung (Kohtala und Bosqué 2014). Auf diese Weise ermöglichen sie beispielsweise Einzelpersonen, aber auch kleinen und mittelständischen Unternehmen (KMU) den Zugang zu innovativen Produktionsverfahren, wie dem 3D-Druck oder dem CNC-Fräsen und vermindern gleichzeitig Opportunitätskosten, die durch Standzeiten verursacht werden.

Anders als (groß-)industrielle Produktionsumgebungen, die in aufwendigen Planverfahren detailliert entworfen und betrieben werden, liegt die Herausforderung von MakerSpaces in der Koordination der unterschiedlichen externen Nutzer, die Prozesse individuell und ohne stetige Wiederholung durchführen. Die so vorherrschende Dynamik führt zu einer nur bedingten Tauglichkeit typischer Strukturmaßnahmen, wie der Reorganisation von Arbeitsprozessen (bspw. Lean Management) oder der Einführung von technischen Lösungen (bspw. Tracking und Automatisierung), die die Planbarkeit des Ressourceneinsatzes deutlich verbessern würden. Das Konzept des DAA erweist sich gegenüber diesen Herausforderungen als robust, da es weder vollständiges Tracking noch bekannte Handlungsabläufe oder Organisationsstrukturen benötigt und Unsicherheiten durch die beschriebene Verfolgung alternativer Lösungsmöglichkeiten abbildet.

Zur Untersuchung der Wirksamkeit des Ansatzes entwickelt CoPDA einen Labordemonstrator, an dem die Einbettung des DAA in einen MakerSpace demonstriert wird. Der Roboter „Mobipick“ (s. Abb. 3) unterstützt hier menschliche Arbeiter bei der Herstellung unterschiedlicher Güter, indem er Werkzeuge und Kleinladungsträger (KLTs), welche Werkstoffe und Werkstücke enthalten, greift und transportiert. Die Herstellung findet in einer Werkstatt an zwei Produktionsinseln statt. Ein Zwischenlager befindet sich in einem Nachbarraum. Einige der menschlichen Arbeiter sind mit einer Augmented-Reality-Brille mit integrierter 3D-Kamera ausgestattet, welche einerseits dem Werker unterstützende Informationen bietet, andererseits vom DAA als zusätzlicher Sensor verwendet wird. Die Arbeiter bewegen sich dabei frei zwischen den Produktionsinseln und anderen Bereichen des MakerSpaces.

In einem Betriebsszenario könnte die Zusammenarbeit zwischen menschlichen und maschinellen Akteuren wie folgt stattfinden:

Ein neuer Nutzer („Nutzer 1“) betritt den leeren MakerSpace, wählt eine Produktionsinsel und holt sich eine Werkzeugkiste aus dem Lager. Kurz darauf betritt ein zweiter Nutzer (,Nutzer 2“) den MakerSpace und bittet den Roboter, seine Werkzeugkiste zur Produktionsinsel zu bringen. Der Roboter findet in diesem Fall durch die Verfolgung unterschiedlicher Hypothesen heraus, ob der Nutzer bereits über eine Werkzeugkiste verfügt. Ist dies der Fall, bringt der Roboter die zugeordnete 
Abb. 3 Der Roboter ,Mobipick" nimmt eine Akku-Bohrmaschine in einer Produktionsstätte auf

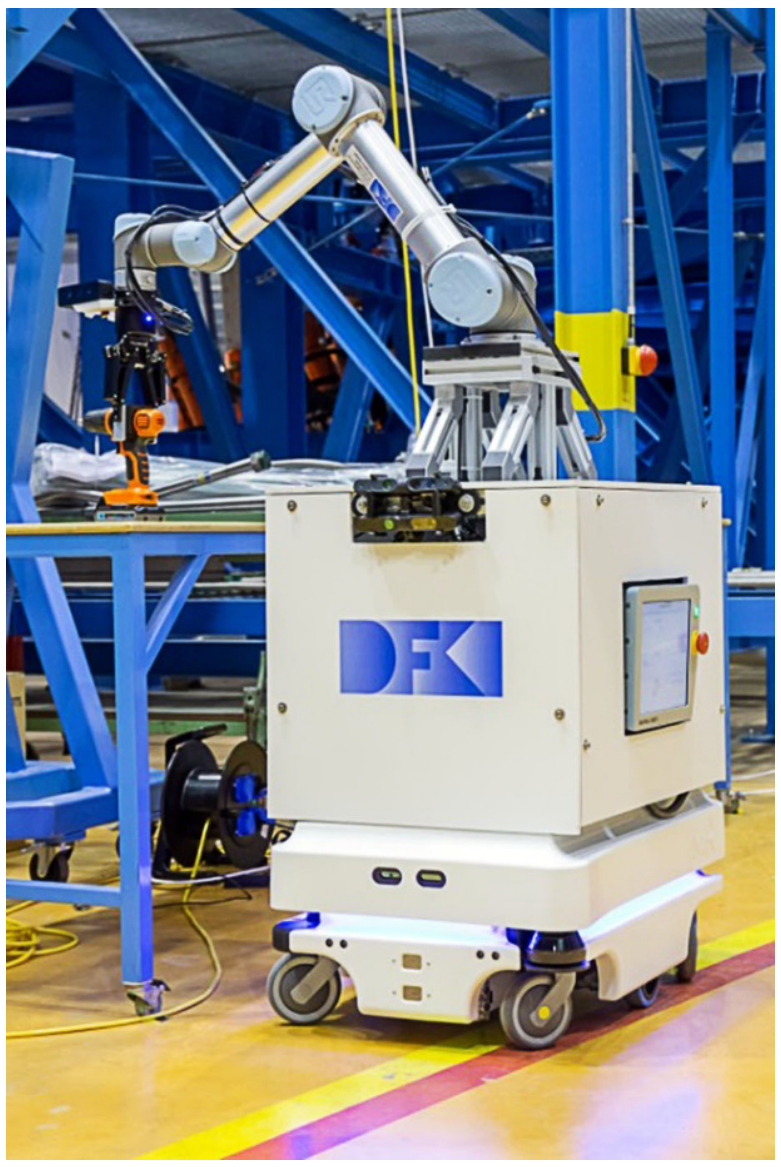

Ausstattung. Besteht bislang keine Zuordnung, soll der Roboter eine bislang nicht genutzte Werkzeugkiste finden und Nutzer 2 bringen. Hierfür greift der Roboter nicht nur auf einen bekannten Werkzeugspeicher zu, sondern identifiziert mittels Objekterkennung auch Werkzeuge, die an anderen Produktionsinseln nicht mehr benötigt werden; beispielsweise, weil der betreffende Nutzer mittlerweile den Raum verlassen und sein Werkstück mitgenommen hat. Während die Nutzer eigenständig Arbeitsschritte durchführen, verfolgt der Roboter eigenständig das Ziel, die Hypothesen im Hypothesenspeicher durch weitere Beobachtungen zu verfeinern und nicht mehr allokierte Ressourcen für die weitere Benutzung freizugeben. Erhält der Roboter weitere Anfragen, werden diese entsprechend des oben genannten Beispiels bearbeitet. Beendet ein Nutzer seine Arbeit, stellt der Roboter den Ausgangszustand einer Produktionsinsel wieder her, indem er zurückgelassene Werkzeuge und Materialien in das jeweilige KLT-Lager bringt. Dabei soll der Roboter beachten, dass Nutzer unter Umständen eigenes, optisch ähnliches Werkzeug oder Material mitbringen könnten und diese Ressourcen nicht in das Lager des MakerSpaces räumen. 


\subsection{Der Hafen als dynamische Outdoor-Umgebung}

Das Hafenszenario implementiert den DAA am Wilhelmshavener Nassauhafen unter ebenfalls unvollständiger Wahrnehmung (nicht alle Bereiche sind durch Sensorerfassung abgedeckt), die durch sich dynamisch verändernden Objekten (z.B. die Gestalt von Segelbooten mit und ohne Segeln) weiter erschwert wird. Anders als der MakerSpace ist die Hafenumgebung zwar in höherem Maße strukturiert, aber dennoch durch hohe Dynamik geprägt. Boote unterschiedlicher Größe und Art, wie etwa Segelboote, Motoryachten oder teilweise auch kleinere Arbeitsschiffe, laufen in einen aus mehreren Bereichen bestehenden Hafen ein und aus, und führen dabei entsprechend notwendige Manöver durch. Dieses Szenario demonstriert den Outdoor Einsatz unter realistischen Umgebungsbedingungen, einschließlich Wettereinflüssen oder unvorhergesehenen menschlichen Handlungen. Eine solche Umgebung ermöglicht darüber hinaus die Untersuchung von Park- und Ladeproblemen im Allgemeinen und deren Bewältigung mit Hilfe des DAA.

Diese und weitere, externe Informationen werden in einer Modelldarstellung verwendet, um prognostische Aussagen zu ermöglichen. Multimodale Sensorinformationen werden von mindestens drei Stellen bereitgestellt, an denen Farb- und Thermalkameras installiert werden (rote Bereiche in Abb. 4a). Ergänzt werden diese durch einen Laserscanner, der Entfernungsinformationen liefert, und Umweltsensoren für die Wetter- und Seebedingungen. Schiffseigene AIS Informationen (Automatic Identification System) werden ebenfalls verwendet, jedoch sind solche Systeme nur für Schiffe mit einer Länge von mehr als 20 Metern vorgeschrieben und daher in kleineren Häfen selten vollständig verfügbar. Anders als der MobiPick-Roboter, der sich im MakerSpace nahezu frei bewegt, verfügen die Kamerasysteme lediglich über eine rudimentäre Steuerung mit Schwenk-, Neige- und Zoomfunktionen. Umgebungsbedingungen werden von einer nahegelegenen Wetterstation und dem allgemeinen Gezeitenkalender geliefert, um den sich ändernden äußeren Bedingungen im Vorhersagemodell Rechnung zu tragen.

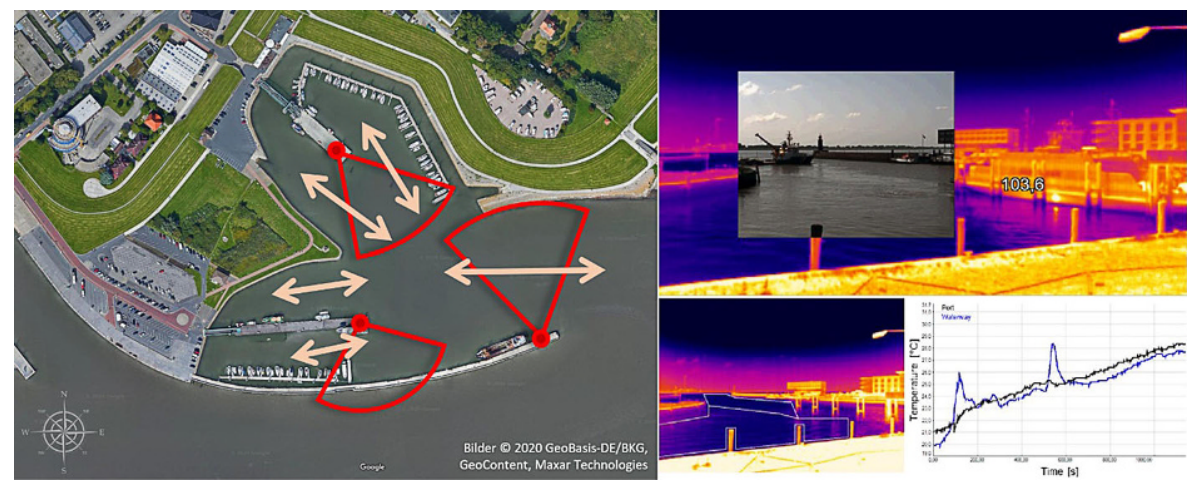

Abb. 4 a Lagebild des Wilhelmshavener Fluthafen (unterer Sektor) und Pontonhafen mit der Nassaubrücke (oberer Sektor). b Detailaufnahmen einzelner Hafenbereiche mittels Wärmebildkamera (farbig) und überlagerter Objekterfassung (s/w) (Rüssmeier et al. 2017) 


\subsection{Anwendungs- und Transferpotenzial}

Typische industrielle Robotikanwendungen planen Einsatzszenarien äußerst rigide voraus, strukturieren erforderliche Abläufe im Detail, um entstehende Dynamiken auf ein Minimum zu reduzieren und überwachen die präzise Ausführung der Planaktivitäten mittels umfassender Tracking-Systeme.

Dem gegenüber erweist sich Anchoring als robuster Ansatz hinsichtlich weniger strukturierbarer Umgebungen und dynamischer Ereignisse, die nicht mit angemessenem Aufwand bis ins letzte Detail vorauszusehen oder zu vermeiden sind. Der Roboter passt sich in die Arbeitsumgebung ein, anstatt die Umgebung dem Roboter anzupassen. Auf diese Weise verringert das Verfahren die praktischen Einstiegsbarrieren in den Einsatz der Robotik, weil Infrastruktur- und Reorganisationsmaßnahmen für die initiale Einführung in einem deutlich geringeren Umfang erforderlich werden und steigert die Eignung von Robotikanwendungen auch für kleine und mittelständische Unternehmen (KMU).

CoPDA entwickelt durch das Object Anchoring einen Schlüsselbaustein, um individualisierte Objekterkennung realisieren zu können. Hierbei handelt es sich in vielen Fällen um eine Grundvoraussetzung, um Mensch-Roboter-Kooperation aus kontrollierten Laborbedingungen in reale Anwendungen überführen zu können. Auf diese Weise entwickelt das Projekt einen Beitrag zur Realisierung der Industrie-4.0-Vision und adressiert gleichzeitig ein international anerkanntes und wichtiges Problem an der Schnittstelle zwischen Künstlicher Intelligenz und Robotik.

Doch welches praktische Potenzial liegt im Dynamic Anchoring für den zukünftigen Robotik-Einsatz? Herauszustellen ist zunächst die Grundabsicht, Anwendungen näher an eine Alltagstauglichkeit heranzuführen und erforderliche Vorarbeiten, wie die Reorganisation und Strukturierung von Arbeitsprozessen sowie Infrastrukturinvestitionen für weitreichende Überwachung abzubauen. In dieser Hinsicht verspricht der Ansatz eine wachsende Robustheit, die in der Praxis bestehende Ressourcenlimitationen mildern könnte. Gerade kleine und mittelständische Unternehmen (KMU), für die der Einsatz von Hochtechnologien in ihrer Produktionsumgebung nicht finanzierbar ist, würden auf diese Weise einen mit weniger Aufwendungen verbundenen Zugang zur Robotik erhalten und in ihrer Innovationsfähigkeit gestärkt werden.

Der Dynamic Anchoring Agent wird damit für Industrieanwendungen relevant, in denen hochautomatisierte oder autonome Maschinen als Kooperationspartner zu menschlichen Mitarbeitern eingesetzt werden und auf hohem semantischem Niveau (z.B. natürliche Sprache) reagieren und interagieren sollen. Gleichzeitig zeigt das zweite Demonstrationsszenario, dass die grundlegende Fähigkeit der Wiedererkennung von Objekten auch außerhalb extensiver Robotikanwendungen neue Potenziale eröffnet. In solchen Fällen wird der DAA als generelleres Werkzeug interpretiert, das lückenhafte Beobachtungen durch das Verfolgen alternativer Hypothesen verbessert und so aktiv zum Informationsmanagement in unstrukturierten und dynamischen Umgebungen eingesetzt werden kann. Der Outdoor-Einsatz am Yachthafen sowie umfangreiche Tests und Validierungen in Zusammenarbeit mit dem lokalen Yachtverein und einem Meeresforschungsinstitut rücken diesen Anwendungsfall in den Mittelpunkt empirischer Untersuchungen, die zukünftiges Anwendungspotenzial auch für weitere Branchen (im Sinne einer „Cross-Innovation“) aufdecken können. 
Funding Open Access funding enabled and organized by Projekt DEAL.

Open Access Dieser Artikel wird unter der Creative Commons Namensnennung 4.0 International Lizenz veröffentlicht, welche die Nutzung, Vervielfältigung, Bearbeitung, Verbreitung und Wiedergabe in jeglichem Medium und Format erlaubt, sofern Sie den/die ursprünglichen Autor(en) und die Quelle ordnungsgemäß nennen, einen Link zur Creative Commons Lizenz beifügen und angeben, ob Änderungen vorgenommen wurden.

Die in diesem Artikel enthaltenen Bilder und sonstiges Drittmaterial unterliegen ebenfalls der genannten Creative Commons Lizenz, sofern sich aus der Abbildungslegende nichts anderes ergibt. Sofern das betreffende Material nicht unter der genannten Creative Commons Lizenz steht und die betreffende Handlung nicht nach gesetzlichen Vorschriften erlaubt ist, ist für die oben aufgeführten Weiterverwendungen des Materials die Einwilligung des jeweiligen Rechteinhabers einzuholen.

Weitere Details zur Lizenz entnehmen Sie bitte der Lizenzinformation auf http://creativecommons.org/ licenses/by/4.0/deed.de.

\section{Literatur}

Bar-Shalom Y, Willett PK, Tian X (2011) Tracking and data fusion-A handbook of algorithms. YBS Publishing, Storrs, CT, USA.

Blodow N, Jain D, Marton Z-C, Beetz M (2010) Perception and probabilistic anchoring for dynamic world state logging. In: Proc. Humanoids 2010. IEEE, Nashville, TN, USA, S 160-166

Blom HAP, Bloem EA (2002) Interacting multiple model joint probabilistic data association, avoiding track coalescence. Proc IEEE Conf Decis Control 3:3408-3415

Coradeschi S, Saffiotti A (2003) An introduction to the anchoring problem. Robot Auton Syst 43:85-96

Coradeschi S, Loutfi A, Wrede B (2013) A short review of symbol grounding in robotic and intelligent systems. Künstl Intell 27:129-136

Deeken H, Wiemann T, Hertzberg J (2019) A spatio-semantic approach to reasoning about agricultural processes. Appl Intell 49:3821-3833. https://doi.org/10.1007/s10489-019-01451-2

Elfring J, van den Dries S, van de Molengraft MJG, Steinbuch M (2013) Semantic world modeling using probabilistic multiple hypothesis anchoring. Robot Auton Syst 61:95-105

Gorecky D, Schmitt M, Loskyll M, Zühlke D (2014) Human-machine-interaction in the industry 4.0 era. In: Proc. 2014 12th IEEE International Conference on Industrial Informatics (INDIN) https://doi.org/ 10.1109/INDIN.2014.6945523

Grenzdörffer T, Günther M, Hertzberg J (2020) YCB-M: a multi-camera RGB-D Dataset for object recognition and 6doF pose estimation. In: Proc. 2020 IEEE International Conference on Robotics and Automation (ICRA), S 3650-3656 https://doi.org/10.1109/ICRA40945.2020.9197426

Günther M, Ruiz-Sarmiento JR, Galindo C, Gonzalez-Jimenez J, Hertzberg J (2018) Context-aware 3D object anchoring for mobile robots. Robot Auton Syst 110:12-32

Harnad S (1990) The symbol grounding problem. Physica D 42:335-346

Kammler F, Brinker J, Vogel J, Hmaid T, Thomas O (2019) How do we support technical tasks in the Age of augmented reality? Some evidence from prototyping in mechanical engineering. In: Proc. International Conference on Information Systems (ICIS 2019)

Kohtala C, Bosqué C (2014) The story of MIT-Fablab Norway: community embedding of peer production. J Peer Prod 5:8

Persson A, Zuidberg Dos Martires P, Loutfi A, De Raedt L (2019) Semantic relational object tracking. IEEE Trans. Cognitive and Developmental Systems, arXiv:1902.09937v1

Pütz S, Simón SJ, Hertzberg J (2018) Move base flex: a highly flexible navigation framework for mobile robots. In: Proc. 2018 IEEE/RSJ International Conference on Intelligent Robots and Systems (IROS), S 3416-3421 https://doi.org/10.1109/IROS.2018.8593829

Reid D (1979) An algorithm for tracking multiple targets. IEEE Trans Automat Contr 24:843-854

Rüssmeier N, Hahn A, Nicklas D, Zielinski O (2017) A research port test bed based on distributed optical sensors and sensor fusion framework for ad hoc situational awareness. J Sens Sens Syst 6:37-52. https://doi.org/10.5194/jsss-6-37-2017 
Tremblay J, To T, Sundaralingam B, Xiang Y, Fox D, Birchfield S (2018) Deep object pose estimation for semantic robotic grasping of household objects. In: Proc. Conference on Robot Learning (CoRL), S 306-316

Wong Lawson LS, Kaelbling LP, Pérez LT (2015) Data association for semantic world modeling from partial views. Int J Robot Res 34(7):1064-1082 